\title{
DESENVOLVIMENTO E COMPOSIÇÃO MINERAL DO PICÃO-PRETO SOB DIFERENTES NÍVEIS DE $\mathbf{p H}^{\mathbf{1}}$
}

\author{
SERGIO Y. OBARA ${ }^{2}$, ALEXANDRE J. BEZUTTE ${ }^{3}$ e PEDRO L. C. A. ALVES
}

RESUMO

\begin{abstract}
Visando estudar a tolerância do picão-preto (Bidens pilosa) $a$ diferentes níveis de $\mathrm{pH}$ do substrato, conduziu-se um ensaio em condições de câmara de crescimento e utilizando-se a técnica de hidroponia, no qual três plântulas foram transplantadas para recipientes contendo solução nutritiva de Hoagland \& Arnon, com pH ajustado para 3,5, 4,0, 5,0, 6,0, 7,0 e 8,0. O delineamento experimental utilizado foi o inteiramente casualizado com três repetições. Ao final do período experimental, 42 dias após o transplante, observou-se que as plantas que se desenvolveram nos pHs 3,5 e 8,0 apresentaram-se menores, com menor comprimento e volume do sistema radicular, menor número de folhas vivas e, consequentemente, menor peso de matéria seca das folhas, caule e raízes. Os teores de $\mathrm{N}$ e $\mathrm{S}$ nas raízes diminuiram com
\end{abstract}

o aumento do $\mathrm{pH}$ do substrato, enquanto os de $\mathrm{Ca}$ e $\mathrm{Mg}$ aumentaram e os de $\mathrm{K}$ e $\mathrm{P}$ diminuiram no $\mathrm{pH}$ 5,0 a 6,0. No caule, apenas o teor de $\mathrm{Mg}$ foi afetado pelo $\mathrm{pH}$, mostrando resposta positiva. $\mathrm{Na}$ folha obteve-se os maiores teores de $\mathrm{P}$, $\mathrm{S}, \mathrm{Ca}$ e $\mathrm{Mg}$ quando a planta se desenvolveu em pH 6,0. Não se constatou diferença significativa entre os efeitos dos $\mathrm{pHs}$ 5,0, 6,0 e 7,0 nos parâmetros avaliados, sendo que foi no $\mathrm{pH}$ 6,0 que se estimou, através de análise de regressão, melhor desenvolvimento do picão-preto. Concluiu-se que o picãopreto apresenta melhor desenvolvimento em $\mathrm{pHs}$ próximo ao neutro, com certa tolerância a pHs extremos $(3,5$ e 8,0).

Palavras-chave: nutrição mineral, biologia, crescimento, planta daninha, Bidens pilosa.

\section{ABSTRACT \\ Development and mineral content of the hairy beggar-ticks under different $\mathrm{pH}$ levels}

An assay was conducted under growth chamber condition to study the hairy beggar-ticks (Bidens pilosa) tolerance to different $\mathrm{pH}$ levels of the substrate. Three seedlings were transplanted into recipients containing Hoaglands \& Anion's nourfishing solution with $\mathrm{pH}$ ajusted to 3.5, 4.0, 5.0, 6.0, 7.0 and 8.0 . The experimental design used was the completely randomized with three replications. At the end of the experimental period (42 days after transplantation) the development of the plants at pHs 3.5 and 8.0 appeared to be smaller in the following aspects: radicular system in length and volume, number of living leaves, and dry matter of the leaves, stem and roots. The $\mathrm{N}$ and $\mathrm{S}$ contents in the roots diminished as the substrate $\mathrm{pH}$ increased, while the $\mathrm{Ca}$ and $\mathrm{Mg}$ contents increased and $\mathrm{K}$ and $\mathrm{P}$ diminished at $\mathrm{pH} 5.0$ to 6.0. $\mathrm{Mg}$ was the only element to be affected in the stem, showing a positive response. The highest contents of $\mathrm{P}, \mathrm{S}, \mathrm{Ca}$ and $\mathrm{Mg}$ were obtained on the leaves when the plant developed at $\mathrm{pH} 6,0$. No significant difference was observed among the $\mathrm{pHs} 5.0$, 6.0 and 7.0 on the evaluated parameters; however, at $\mathrm{pH} 6.0$ a better development of the beggar-tick was evaluated through a regression analysis. It was concluded that the beggar-tick shows its better development at pHs close to neutral, although it presents certain tolerance to extreme $\mathrm{pHs}$ (3.5 and 8.0).

Additional index words: mineral nutrition, weed biology, plant growth, weed, Bidens pilosa.

1 Recebido para publicação em 11/06/93 e na forma revisada em 20/01/94.

2 Eng $^{\circ}$ Agr $^{\circ}$, Aluno de Pós-graduação, ESALQ/USP, Depto. de Horticultura, 13418-900 Piracicaba, SP

3 Eng $^{\circ}$ Agr $^{\circ}$, Bolsista de Aperfeiçoamento,Depto. de Biologia Aplicada, FCAV/UNESP, 14870-000 Jaboticabal, SP

4 Prof. Assistente, Depto.de Biologia Aplicada, FCAVIUNESP, 14870 -000 Jaboticabal, SP 


\section{INTRODUÇÃO}

Dentre os fatores que influenciam o potencial produtivo das espécies cultivadas, pode-se destacar as plantas daninhas que, quando presentes em ecossistemas agrícolas causam reduções quantitativas e qualitativas na produção.

O picão-preto (Bidens pilosa L.), está incluído entre as principais espécies daninhas em vários países do mundo (Holm et al, 1991). No Brasil, esta espécie encontra-se distribuída por todo o pais, principalmente nas regiões Centro-sul e Sul, onde é considerada uma espécie altamente nociva e séria infestante de culturas anuais e perenes.

Segundo Tamashiro \& Leitão (1978), o picão-preto reúne quase todos os aspectos morfológicos e biológicos que a caracterizam como invasora típica: crescimento rápido, porte herbáceo, ocorrência de várias gerações durante o ano, alta produção de sementes com elevada viabilidade, eficiente mecanismo de dispersão de frutos e, ainda, produção de frutos polimórficos, o que favorece a adaptação a ambientes variados.

Devido às alterações que vem sofrendo o conceito de plantas daninhas, às novas técnicas empregadas visando o seu controle e, principalmente, à introdução do manejo integrado, são de fundamental importância os estudos básicos de biologia das espécies de ocorrência comum nos agroecossistemas brasileiros. $\mathrm{O}$ crescimento de cada indivíduo, em sua maior parte, não é determinada pelo potencial genético da espécie e sim pela disponibilidade de recursos e capacidade de adaptação em um ambiente extremamente concorrido.

A habilidade competitiva relativa de espécies de plantas daninhas e de culturas varia com o pH do solo (Weaver \& Hamill 1985). Pela importância do picão-preto como espécie daninha e pela necessidade de novos conhecimentos sobre o seu comportamento e sua adaptação, objetivou-se, neste trabalho, avaliar os efeitos do $\mathrm{pH}$ sobre o seu desenvolvimento e composição mineral (macroelementos).

\section{MATERIAL E MÉTODOS}

No presente trabalho utilizou-se a técnica de hidroponia, sendo este conduzido sob condições de câmara de crescimento, ajustada para temperatura de $25^{\circ} \mathrm{C}$ (1), fotoperíodo de 14 horas e intensidade luminosa de $108 \mathrm{uE} \cdot \mathrm{m}^{-2} \cdot \mathrm{s}^{-1}$.

Aquênios de $B$. pilosa foram colocados para germinar em substrato de areia de rio, lavada e esterilizada $\left(120^{\circ} \mathrm{C}\right.$ por 96 horas). Quando as plantas apresentavam dois pares de folhas totalmente expandidas, foram selecionadas quanto à uniformidade de tamanho e transferidas em número de três por recipiente. Nesta fase de adaptação, plantas mortas foram substituídas por novas plantas.

Os recipientes constaram de vasos plásticos com capacidade para 4,5 litros. O substrato de crescimento das plantas constou de solução nutritiva de Hoagland \& Arnon (1950), com a concentração ajustada conforme a idade da planta e o $\mathrm{pH}$ ajustado de acordo com o tratamento experimental. Nas primeiras duas semanas, a solução nutritiva foi diluída em $50 \%$, nas duas semanas seguintes foi diluída para $75 \%$, quando então foi utilizada a concentração original, proposta pelos autores, até o término da condução do trabalho, com trocas semanais da solução. Para obtenção e manutenção dos diferentes níveis de $\mathrm{pH}$, correspondentes aos tratamentos, foram adicionad as à solução nutritiva quantidades de hidróxido de sódio $(\mathrm{NaOH})$ 0,1 $\mathrm{N}$ ou ácido clorídrico $(\mathrm{HCl})$ $0,1 \mathrm{~N}$ suficientes para aumentar ou diminuir o $\mathrm{pH}$, sendo a aferição realizada diariamente por meio de potenciômetro.

$\mathrm{O}$ delineamento experimental utilizado foi o inteiramente casualizado com três repetições e os tratamentos constaram dos valores de $\mathrm{pH}$, a saber: 3,5, 4,0, 5,0, 6,0, 7,0 e 8,0 . Os dados referentes aos parâmetros de crescimento foram submetidos à análise de variância pelo teste $\mathrm{F}$, e a comparação das médias pelo teste de Tukey, seguidas de análises de regressão polinomial.

Ao término do período experimental, 42 dias após a transferência das plantas, os parâmetros avaliados foram: número de folhas vivas e mortas, altura da planta, comprimento da raiz principal, volume radicular (calculado por deslocamento de volume de água), número de ramos e acúmulo de matéria seca em folhas, caule + ramos e raízes. Adotou-se 42 dias de condução pois, segundo Michelini (1983), a fase de crescimento mais intenso de B. pilosa é de 28 aos 42 dias após a emergência, quando este mais extrai os recursos do meio e, portanto, apresenta maior potencial competitivo. A matéria seca das diferentes partes da planta foi obtida após secagem do material em estufa com circulação forçada de ar, a $70-80^{\circ} \mathrm{C}$ por 96 horas. Depois de seco e pesado, o material vegetal foi moído em micromoinho tipo Willey com peneira de 60 "mesh". Do material moído coletaram-se amostras para serem submetidas às digestões sulfúrica e nitroperclórica e no digerido determinaram-se os teores de N, P, K, Ca, $\mathrm{Mg}$ e $\mathrm{S}$.

Os teores de $\mathrm{N}$ total foram determinados pelo método semi-micro Kjeldahl; teores de $\mathrm{P}$ pelo método colorimétrico do ácido fosfovanadatomolibdico e os teores de $\mathrm{K}, \mathrm{Ca}$, e $\mathrm{Mg}$ pela espectrofotometria de absorção atômica, todos descritos em Sarruge \& Haag (1974). O S foi determinado pelo método turbidimétrico, descrito em Vitti (1989). Essas análises foram realizadas no Laboratório Central da FCAVJ/ UNESP, localizado emJaboticabal, SP.

\section{RESULTADOS E DISCUSSÃO}

Plantas de Bidens pilosa que se desenvolveram em pHs 3,5 e 8,0 mostraram-se menores quando comparadas às que se desenvolveram em níveis de $\mathrm{pH}$ variando de 4,0 a 7,0 (Tabela 01). Quando se realizou a análise de regressão polinomial deste parâmetro eni função dos níveis de $\mathrm{pH}$ da solução, obteve-se um comortamento quadrático $(\mathrm{Y}=$ $\left.-113,54+50,12 X-4,21 X^{2} ; 11^{2}=85,01^{* *}\right)$, com estimativa da altura máxima das plantas em pH 6,0.

Quando se avaliou o sistema radicular das plantas, observou-se que aquelas que se desenvolveram em $\mathrm{pHs} 3,5 \mathrm{e}$ 4,0 apresentaram red uções no comprimento das raízes, quando comparadas com as plantas que se desenvolveram entre níveis de $\mathrm{pH}$ variando de 5,0 a 8,0. Já com relação ao volume radicular a redução foi mais drástica somente em $\mathrm{pH}$ 3,5 (Tabela 1). Submetendo estes valores a análise de regressão polinomial em função dos níveis de $\mathrm{pH}$ da solução, obteve-se para o comprimento radicular um comportamento 
S.Y. Obara et al.

TABELA 1 - Valores médios da altura da planta, volume e comprimento do sistema radicular, número de folhas vivas, folhas mortas e ramos de Bidens pilosa, além do respectivo peso de matéria seca em folhas, raízes e ramos, avaliadas aos 42 DAT. Jaboticabal, 1991.

\begin{tabular}{|c|c|c|c|c|c|c|c|c|c|}
\hline \multirow{2}{*}{$\begin{array}{l}\text { Tratamentos } \\
\text { Níveis de } \\
\text { (pH) }\end{array}$} & \multirow{2}{*}{$\begin{array}{c}\text { Altura da } \\
\text { Planta } \\
\text { (cm) }\end{array}$} & \multicolumn{2}{|c|}{ Sistema Radicular } & \multicolumn{3}{|c|}{ Numero por Planta ${ }^{(1)}$} & \multicolumn{3}{|c|}{ Matéria Seca (g) } \\
\hline & & $\begin{array}{c}\text { Compri- } \\
\text { mento }(\mathrm{cm})\end{array}$ & $\begin{array}{c}\text { Volume } \\
\left(\mathrm{cm}^{3}\right)\end{array}$ & Fls. Vivas & $\begin{array}{c}\text { Fls. } \\
\text { Mortas }\end{array}$ & Ramos & Folhas & Raízes & Caule \\
\hline 3,5 & $5,14 \mathrm{~d}^{(2)}$ & $3,77 \mathrm{~d}$ & $0,29 \mathrm{c}$ & $3,15 \mathrm{~d}$ & $1,77 \mathrm{a}$ & $0,71 \mathrm{c}$ & $0,22 \mathrm{~d}$ & $0,05 \mathrm{c}$ & $0,05 \mathrm{e}$ \\
\hline 4,0 & $26,87 \mathrm{~b}$ & $48,88 \mathrm{c}$ & $10,50 \mathrm{bc}$ & $8,24 \mathrm{~b}$ & $1,76 \mathrm{a}$ & $3,38 \mathrm{~b}$ & $4,37 \mathrm{~b}$ & $0,97 \mathrm{~b}$ & $1,15 \mathrm{c}$ \\
\hline 5,0 & $31,67 \mathrm{ab}$ & $77,30 \mathrm{ab}$ & $19,92 \mathrm{~b}$ & $9,34 \mathrm{a}$ & $1,56 \mathrm{a}$ & $4,11 \mathrm{a}$ & $6,73 \mathrm{a}$ & $1,62 \mathrm{a}$ & $2,21 \mathrm{a}$ \\
\hline 6,0 & 33,50 a & $68,72 \mathrm{ab}$ & 34,67 a & 9,23 a & 1,91 a & $4,52 \mathrm{a}$ & $4,94 \mathrm{~b}$ & 1,56 a & $1,81 \mathrm{ab}$ \\
\hline 7,0 & $30,32 \mathrm{ab}$ & $61,92 \mathrm{bc}$ & 24,67 ab & $8,49 \mathrm{~b}$ & 1,65 a & 4,11 a & $5,65 \mathrm{ab}$ & 1,72 a & $1,71 \mathrm{~b}$ \\
\hline 8,0 & $19,18 \mathrm{c}$ & 78,75 a & $17,42 \mathrm{~b}$ & $7,07 \mathrm{c}$ & 1,82 a & $3,44 \mathrm{~b}$ & $2,62 \mathrm{c}$ & $0,83 \mathrm{~b}$ & $0,55 \mathrm{~d}$ \\
\hline F trat. & $44,71^{* *}$ & $32,69^{* *}$ & $7,41^{* *}$ & $127,04^{* *}$ & $0,70^{\text {ns }}$ & $66,47^{\circ *}$ & $21,53^{* *}$ & $30,45^{* *}$ & $34,13^{* *}$ \\
\hline $\mathrm{CV}(\%)$ & 11,36 & 15,05 & 41,76 & 4,70 & 14,86 & 8,67 & 21,35 & 17,85 & 19,63 \\
\hline
\end{tabular}

* - significativo ao nível de $1 \%$ de probabilidade pelo teste $\mathrm{F}$.

ns - nāo significativo ao nível de $1 \%$ de probalidade pelo teste $F$.

(1) - Para a análise de variància os dados foram transformados para $\sqrt{\mathrm{X}+0,50}$.

(2) - Médias seguidas pela mesma letra não difere entre sí pelơ teste de Duncan a $5 \%$ de probabilidade.

cúbico ( $\mathrm{Y}=-1159,77+633,13 \mathrm{X}-106,02 \mathrm{X}^{2}+5,78 \mathrm{X} 3 ; \mathrm{R}^{2}=$ 99,53 ), demonstrando que apenas pHs mais ácidos reduzem o comprimento da raiz. Para o volume radicular foi obtido um comportamento quadrático $(\mathrm{Y}=-126,40+50,23 \mathrm{X}-$ $\left.4,04 X^{2} ; R^{2}=93,04 * *\right)$ onde o valor máximo foi obtido em pH 6,2, demonstrando que pHs extremos afetam igualmente o volume radicular.

Plantas de B. pilosa que se desenvolveram em solução nutritiva com $\mathrm{pH} 3,5,4,0$ e 8,0 mostravam-se menos ramificadas que aquelas que se desenvolveram em $\mathrm{pH}$ de 5,0 a 7,0. A análise de regressão polinomial revelou comportamento quadrático $\left(\mathrm{Y}=-12,96+5,71 \mathrm{X}-0,46 \mathrm{X}^{2} ; \mathrm{R}^{2}=85,19^{* *}\right)$, com ramificação máxima da planta estimada com pH 6,2.

Quando avaliou-se o número de folhas vivas, verificouse que as plantas que se desenvolveram em pHs 5,0 e 6,0 apresentaram aumento neste parâmetro quando comparadas às que se desenvolveram nos demais níveis de $\mathrm{pH}$, sendo que aquelas que se desenvolveram em pHs 3,5 e 8,0 mostraramse com menor número de folhas vivas quando comparadas às que se desenvolveram em pHs 4,0 e 7,0. A análise de regressão polinomial mostrou comportamento quadrático para este parâmetro $\left(Y=-20,36+10,04 X-0,83 X^{2} ; R^{2}=76,8 * *\right)$, com valor máximo estimado para o $\mathrm{pH}$ 6,0. Os níveis de $\mathrm{pH}$ da solução não afetaram a taxa de mortalidade das folhas, demonstrando que a redução no número de folhas em função do $\mathrm{pH}$ foi devida à menor taxa de emissão destas.

Os pesos da matéria seca das raízes e do caule das plantas de B. pilosa que se desenvolveram em $\mathrm{pHs} 3,5 ; 4,0 \mathrm{e}$ 8,0 (Tabela 1) apresentaram reduções significativas quando comparadas às plantas que se desenvolveram em níveis de $\mathrm{pH}$ variando de 5,0 a 7,0. Com a realização da regressão polinomial em função da variação dos níveis de $\mathrm{pH}$ da solução, observou-se que estes parâmetros apresentaram um comportamento quadrático, onde o peso da matéria seca das raízes $\left(Y=-7,29+3,02 X+-0,25 X^{2} ; R^{2}=92,58^{* *}\right)$ apresen- tou valor máxi mo estimado em $\mathrm{pH} 6,0$, e do caule $(\mathrm{Y}=$ $\left.-8,80+3,71 \mathrm{X}-0,32 \mathrm{X}^{2} ; \mathrm{R}^{2}=80,79^{* *}\right)$ em pH 5,8 .O peso da matéria seca das folhas apresentou reduções mais acentuadas nas plantas que se desenvolveram em pH 3,5 e 8,0 e a análise de reg ressão demonstrou um comport amento quádrático $\left(\mathrm{Y}=-24,08+10,24 \mathrm{X}-0,86 \mathrm{X}^{* *} ; \mathrm{R}^{2}=63,07 * *\right)$ onde o peso máximo foi estimado em $\mathrm{pH} 6,0$.

Obara \& Alves (1990), analisando os efeitos do $\mathrm{pH}$ sobre o desenvolvimento de $B$. pilosa em solo, verificaram que as plantas que se desenvolveram em $\mathrm{pH}$ 6,5 e 7,0 apresentaram-se mais altas e com o volume radicular maior do que as demais ( $\mathrm{pH} 4,0$ a 6,0), embora não diferissem no comprimento do sistema radicular e no número de folhas. Observaram também que nestas plantas (pH 6,5 e 7,0) o peso da matéria seca da parte aérea e do sistema radicular foi maior.

Quando se analisou os teores de macroelementos nas raízes de $B$. pilosa submetidas a diferentes níveis de $\mathrm{pH}$ (Figura 1) observou-se que, para nitrogênio $(\mathrm{N})$, com o aumento do $\mathrm{pH}$ houve redução na sua concentração. $\mathrm{O}$ pH da solução entre 5,0 e 6,0 reduziu o teor de fósforo $(\mathrm{P})$ nas raízes. Para o enxofre (S) verificou-se que, a partir do $\mathrm{pH} 4,0$, houve redução no seu teor nas raízes. O teor de potássio (K) foi menor nas raízes que se desenvolveram em pH 5,0 e 6,0, quando comparado aos valores extremos de $\mathrm{pH}$. 0 acúmulo de cálcio (Ca) e magnésio $(\mathrm{Mg})$ nas raízes foi crescente com $\mathrm{o}$ aumento do $\mathrm{pH}$.

Os teores de N, P, S e Ca no caule da planta de picão-preto não foram influenciados pelo $\mathrm{pH}$ da solução, enquanto o de $\mathrm{K}$ decresceu nos pHs 5,0 e 6,0 e o de $\mathrm{Mg}$ aumentou até $\mathrm{pH} 7,0$, após o qual ficou constante.

Os teores de N, P e K foram menores nas folhas quando as plantas se desenvolveram em solução com $\mathrm{pH} 3,5$, enquanto os de $\mathrm{S}, \mathrm{Ca}$ e $\mathrm{Mg}$ foram maiores. No $\mathrm{pH}$ 4,0 foram observados os maiores teores de $\mathrm{N}$ e $\mathrm{K}$ nas folhas, após o 


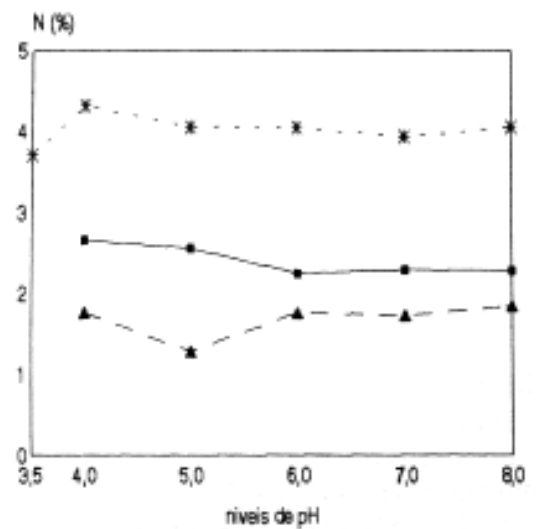

- raizes * caule * folhas

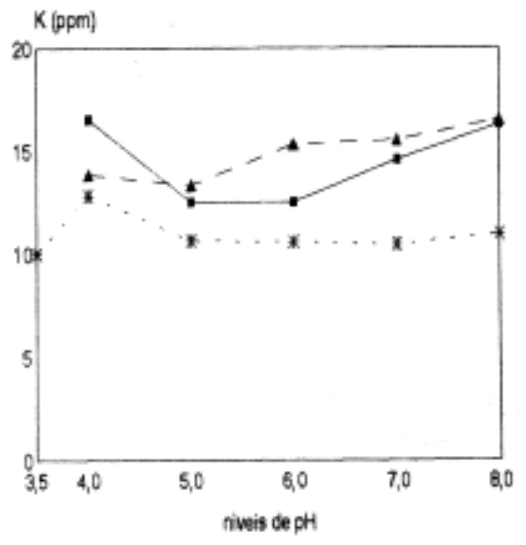

- razes * cade Ffolhas

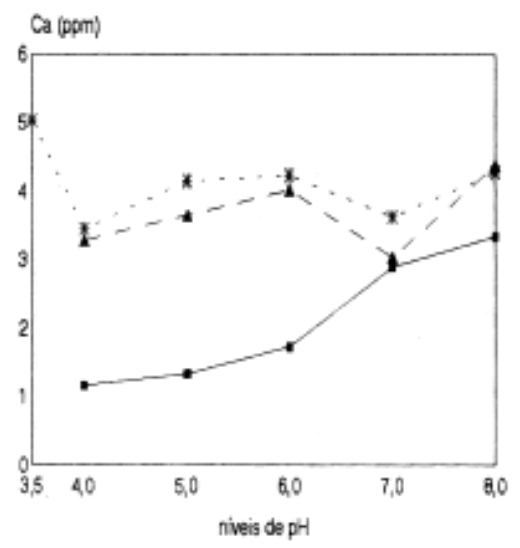

- razes * cade * iohas

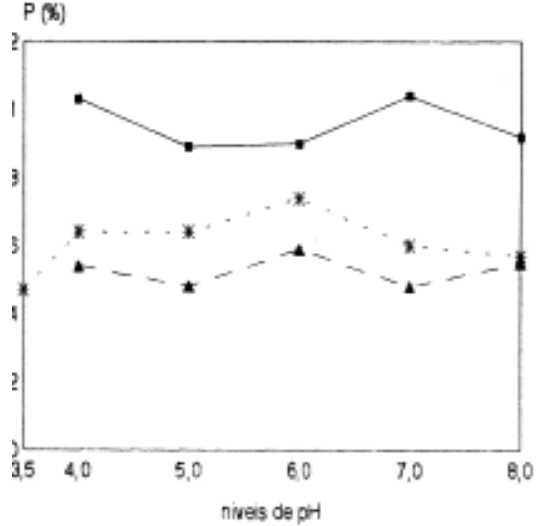

- razes * caule * tolhas

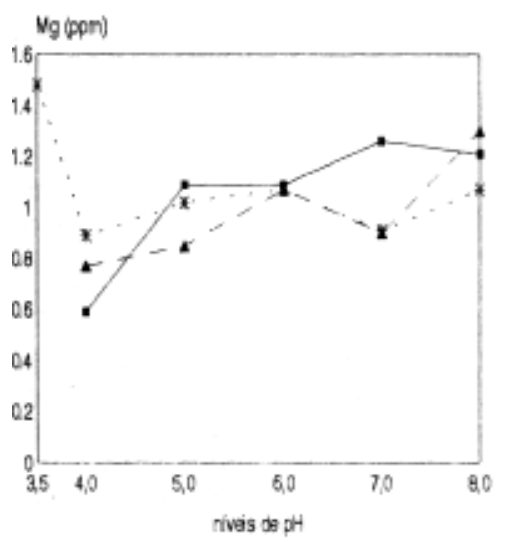

- razes * caule *ichas

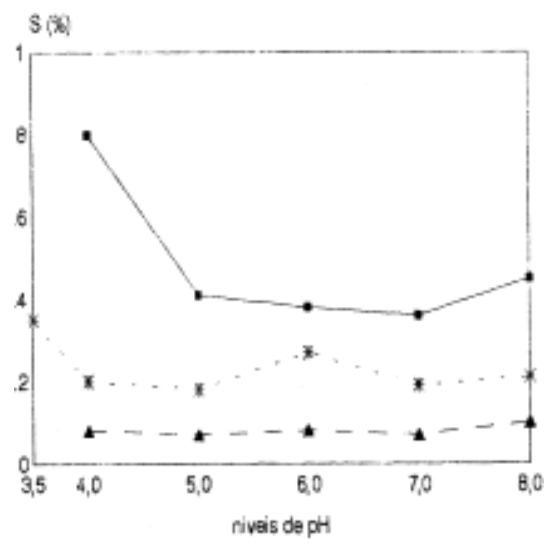

- raizes * caule * fohas

FIG. 1 - Teores de macroelementos nas raízes, caules e folhas de Bidens pilosa, sob efeito de diferentes níveis de pH, Jaboticabal, SP, 1991.

qual ( $\mathrm{pH} 5,0$ a 8,0) estes mantiveram-se constantes, enquanto no $\mathrm{pH}$ 6,0 verificou-se aumento nos teores de $\mathrm{P}, \mathrm{S}, \mathrm{Ca}$ e $\mathrm{Mg}$.

$\mathrm{O} \mathrm{pH}$ do substrato proporciona efeitos diretos e indiretos na planta (Malavolta, 1980). Como efeito direto tem-se a concentração hidrogênica atuando sobre a planta e, como indireto, tem-se a concentração hidrogênica atuando sobre a disponibilidade de elementos no substrato que, por sua vez, afetará a planta. No caso de se utilizar solução nutritiva como substrato de crescimento, tem-se apenas o efeito da concentração hidrogênica atuando sobre a planta.

Considera-se que a disponibilidade não é influenciada pelos tratamentos, ou seja, todos os elementos são mantidos disponíveis em solução, em concentrações adequadas ao crescimento, diferentemente de ensaios de $\mathrm{pH}$ conduzidos 
em solo. Como efeitos diretos, Malavolta (1980) relata que o tomateiro apresenta crescimento máximo quando o $\mathrm{pH}$ da solução é mantido entre 6,0 e 6,5 , sendo que em valores extremos de $\mathrm{pH}(3,0$ e 9,0) as plantas morrem; a análise mineral das plantas revelou que $\mathrm{pH}$ muito baixo prejudicou a absorção de $\mathrm{N}, \mathrm{K}, \mathrm{P}$ e Ca, sendo que em pH 3,0 as plantas perderam $\mathrm{P}$ e Ca para a solução; quando o $\mathrm{pH}$ é maior do que 7,0 diminui a absorção de N, $\mathrm{P}$ e Ca.

Comparando-se os efeitos do pH 4,0 a 8,0 da solução na absorção e, consequentemente, na concentração dos macroelementos na planta inteira de $B$. pilosa, observou-se que em $\mathrm{pH}$ 4,0 houve men or absorção de $\mathrm{Ca}$ e $\mathrm{Mg}$ e maior absorção de $\mathrm{K}$ e $\mathrm{S}$, sem afetar a de $\mathrm{N}$ e $\mathrm{P}$.

Neste ensaio, independente do pH da solução, observou-se que as folhas apresentaram a maior concentração de $\mathrm{N}$ e $\mathrm{Ca}$; as raízes de PeSeo caule de K. A concentração de $\mathrm{Mg}$ praticamente se igualou em todas as partes da planta.

Costallat (1974), estudando em solo a absorção de nutrientes por $B$. pilosa, verificou que o $\mathrm{K}$ foi absorvido em maiores quantidades, seguindo-se o $\mathrm{N}$ e, em menor escala, o $\mathrm{P}$ e o $\mathrm{Mg}$. A maior concentração de $\mathrm{N}$, aos 65 dias após a emergência, ocorreu nas folhas, enquanto para o P, K e Mg o maior acúmulo se deu no caule. Michelini (1983), estudando a absorção e distribuição de nutrientes em $B$. pilosa cultivada em solo, observou que aos 70 dias após a emergência, as maiores quantidades dos macroelementos sempre eram encontradas nas folhas, seguidas do caule e das raízes. Para o N e $\mathrm{P}$, os teores nas raízes foram maiores do que no caule, enquanto para $\mathrm{K}, \mathrm{Ca}$ e $\mathrm{Mg}$ ocorreu o contrário.

Costallat (1974) observou que B. pilosa absorveu com maior intensidade os nutrientes do solo no período de 50 a 80 dias após a emergência, fase de pleno florescimento e frutificação. Michelini (1983) verificou que o maior acúmulo de $\mathrm{N}$ na planta ocorreu aos 56 dias, enquanto os de matéria seca, $\mathrm{P}$, $\mathrm{K}$, Ca e Mg ocorreram aos 70 dias após a emergência.

São poucos os estudos sobre o efeito dos diferentes níveis de $\mathrm{pH}$ do solo ou da solução nutritiva sobre o desenvolvimento e a nutrição mineral de plantas daninhas. Os baixos valores de $\mathrm{pH}$ são desfavoráveis ao estabelecimento de Eleocharis parvula (Bissel \& Frank, 1980). Em Solidago altissima, Rani \& Hijimoto (1981) observaram que os teores de $\mathrm{K}, \mathrm{Ca}$ e $\mathrm{Mg}$ eram acumulados à medida que se aumentava $\mathrm{o}$ $\mathrm{pH}$, embora, em termos de crescimento, a planta mostrasse adaptação às variações de $\mathrm{pH}$ do solo. Zaprzalka \& Peters (1982), encontraram pequena variação no crescimento de Taraxacum officinale, nos valores de $\mathrm{pH}$ do solo de 4,7, 6,7 e 7,6 .

$\mathrm{O}$ efeito do $\mathrm{pH}$ do solo entre os níveis de 3,4 e 7,8, sobre os teores de nutrientes de $B$. pilosa encontrou correlação positiva no aumento do $\mathrm{pH}$ com as concentrações de $\mathrm{Ca}$ e $\mathrm{Mg}$ nas raízes, sendo que em $\mathrm{pH} \mathrm{7,8}$ houve decréscimo (Michelini, 1983). A concentração de $\mathrm{K}$ nas raízes diminuiu com o aumento do $\mathrm{pH}$, enquanto a de $\mathrm{P}$ não foi alterada. Com relação a parte aérea da planta, a variação do $\mathrm{pH}$ do solo não alterou significativamente os teores de $\mathrm{P}$ e Ca; porém, com o aumento do $\mathrm{pH}$ os teores de $\mathrm{Mg}$ cresceram linearmente, enquanto os de $\mathrm{K}$ decresceram.

Pavani (1992), estudando o crescimento e a nutrição de Cenchrus echinatus a diferentes níveis de $\mathrm{pH}(3,5$ a 7,0$)$ em solução nutritiva, observou que todas as partes da planta responderam positivamente ao aumento do $\mathrm{pH}$, conseguindo considerável crescimento em valores de $\mathrm{pH}$ muito baixos. Verificou ainda que os teores de $\mathrm{N}$ total nas folhas e nos caules+bainhas e os teores de $\mathrm{Mg}$ e $\mathrm{S}$ nos caules+bainhas e na média global da planta sofreram rápidos decréscimos desde $\mathrm{pH}$ 3,5 até $\mathrm{pH}$ 6,0 e pH 5,0, respectivamente, e depois tenderam à estabilização. Os teores de $\mathrm{Ca}$ e $\mathrm{Mg}$ no sistema radicular cresceram com o $\mathrm{pH}$ da solução. Nas folhas, o teor de $\mathrm{Mg}$ cresceu linearmente com o aumento do $\mathrm{pH}$, enquanto o de Ca não se alterou. Os teores de $\mathrm{K}$ nas raízes, de $\mathrm{P}, \mathrm{Ca}$ e $\mathrm{Mg}$ nos caules + bainhas e de $\mathrm{S}$ nas folhas não foram influenciados pelo $\mathrm{pH}$.

\section{LITERATURA CITADA}

BISSEL, S.R.; FRANK, P.A. Soil factors that influence the establishment and growth of dwart spiderush (Eleocharis parvula (R\&S) Link). Weed Abstracts, Wallingford, v.29, n.6, p.1702, 1980.

COSTALLAT, R.F. Absorção de nutrientes e sua distribuição em Bidens pilosa L. Jaboticabal: F.C.A.V., 1974. 28p. Trabalho de Graduação em Agronomia.

HOAGLAND, D.R.; ARNON, D.I. The water culture method of growing plants without soil. Berkeley: University of California, 1950. 20p.(Circular 347).

HOLM, Le R.G.; PLUCKNETT, D.L; PANCHO, J.V.; HERBERGER, J.P. The word's worst weeds: distribution and biology. Honolulu: East West Food Institute, 1991. p.185-190.

ITANI, T.; HIJIMOTO, S. Ecological characteristics of Solidago altissima L. 2.Effects of nitrogen, phosphorus and calcium levels on growth. Bulletin of the Hiroshima Agricultural College, Hiroshima, v.6, n.4, p.373-378, 1981.

MALAVOLTA, E. Elementos de nutrição mineral de plantas. São Paulo: Ed. Agronômica Ceres, 1980. p.2741.

MICHELINI, L.A. Nutrição mineral de plantas daninhas: absorção e distribuição de nutrientes em Bidens pilosa L. e Brachiaria plantaginea (Link) Hitch e efeito do $\mathrm{pH}$ do solo nos teores de nutrientes em Bidens pilosa L. e Echinochloa colonum (L.) Link. Jaboticabal: F.C.A.V., 1983. 83p. Trabalho de Graduação em Agronomia.

OBARA, S.Y.; ALVES, P.L.C.A. Efeito do pH do solo sobre o desenvolvimento de picão preto. In: CONGRESSO DE INICIAÇÃO CIENTIFICA, 2., 1990, Botucatu. Resumos...p.183.

PAVANI, M.C.M.D. Estudos sobre o crescimento, nutrição mineral de Cenchrus echinatus L. e resposta a diferentes níveis de fósforo, pH e calagem. Jaboticabal: F.C.A.V., 1992. 129p. Dissertação de Doutorado em Agronomia.

SARRUGE, J.R.; HAAG, H.P. Análises químicas em plantas. Piracicaba: ESALQ, 1974. 56p. Mimeografado.

TAMASHIRO, S.Y.; LEITÃO FILHO, H.F. Observações sobre o cíclo de vida de Bidens pilosa (Compositae, Heliantheae). Hoehnea, v.7, p.27-40, 1978.

V1TTI, G.C. Avaliação e interpretação do enxofre no solo e na planta. Jaboticabal: FUNEP, 1989. 37p.

WEAVER, S.E.; HAMILL, A.S. Effects of soil $\mathrm{pH}$ on competitive ability and leaf nutrient content of corn (Zea mays L.) and three weed species. Weed Science, Champaign, v.33, n.4, p.447-451, 1985. ZAPRZALKA,

J.R.; PETERS, R.A. Growth of dandelion as influenced by lime and fertility levels. In: NORTHEASTERN WEED SCIENCE SOCIETY, s/1, 1982. Proceedings... p.29-32. 